\title{
TOTAL NUMBER OF JUMP-POINTS OF ORDERED PURE $P$-EXTENSIONS
}

\section{WEN AN LIU and JIN LU WANG}

College of Mathematics and Information Science

Henan Normal University

Xinxiang 453007

P. R. China

e-mail: liuwenan@126.com

\begin{abstract}
Fraenkel and Tanny in [9] introduced Wythoff-like games, denoted by Wyt $(f)$. We define a class of new games based on a given $\operatorname{Wyt}(f)$ : Let $\Gamma_{K}$ be the new game obtained from $\mathrm{Wyt}(f)$ by adjoining to it the first $K P$-positions as additional moves. For an integer $m \geq 1$, if the set of all $P$-positions of $\Gamma_{m}$ does not equal to the set of all $P$-positions of $\Gamma_{m-1}$, we call $m$ a jump-point of $\operatorname{Wyt}(f)$.

The main purpose of this paper is to analyze the structure and total number of jump-points of Wyt $(f)$ for $f(x)=s x$ with integer coefficient $s \geq 2$. It turns out that 1 is the only jump-point if $s=2$, and 1 and 2 are the only two jump-points if $s \geq 3$.
\end{abstract}




\section{Introduction}

By game we mean a combinatorial game; we restrict our attention to classical impartial games. There are two conventions: in normal play convention, the player first unable to move is the loser (his opponent the winner); in misère play convention, the player first unable to move is the winner (his opponent the loser). The positions from which the previous player can win regardless of the opponent's moves are called P-positions, and those from which the next player can win regardless of the opponent's moves are called $N$-positions. See [1, 2].

Wythoff's game is played with two piles of tokens. Each player can either remove any positive number of tokens from a single pile (Nim-rule) or remove the same positive number of tokens from both piles (Wythoffrule). All $P$-positions of Wythoff's game under normal play convention were given in [12]. All $P$-positions of Wythoff's game under misère play convention were determined in [7].

Given a game $\Gamma$, let $\mathscr{M}(\Gamma)$ be the set of all moves of $\Gamma$ and $\mathscr{P}(\Gamma)$ be the set of all $P$-positions of $\Gamma$. If $\mathscr{M}(\Gamma) \subset \mathscr{M}\left(\Gamma_{1}\right)$, we call $\Gamma_{1}$ an extension of $\Gamma$. In many papers devoted to variations of Wythoff's game, new rules are adjoined to the original ones. In particular, the following extensions can be found in the literature.

\subsection{Wythoff-like games}

Fraenkel in [5] introduced the so-called $(s, t)$-Wythoff's game, where the Wythoff-rule is changed into the More General Wythoff-rule: Take tokens from both piles, $k>0$ from one pile and $\ell>0$ from the other, such that $0<k \leq \ell<s k+t$. Under normal or misère play convention, all $P$-positions of $(s, t)$-Wythoff's game were given in $[5,10]$ for all integers $s$, $t \geq 1$.

Fraenkel and Tanny in [9] defined an even more general version of the game by replacing $s k+t, s, t>0$, by an arbitrary function $f: \mathbb{Z}_{\geq 0} \rightarrow \mathbb{Z}_{\geq 0}$ and requiring that $0<k \leq \ell<f(k)$, the Wyt $(f)$-rule. Hence, for any function $f$, one has a Wythoff-like game, denoted by Wyt $(f)$. 


\subsection{Pure $P$-extension}

It was pointed out in Section 6 of [4] that interesting games can be obtained by adjoining to a given game an appropriate subset of its $P$-positions as moves. Such an extension is called to be pure P-extension, i.e., all additional moves are $P$-positions. For example, Wythoff's game is 2-pile Nim with all $P$-positions adjoined. The idea was also exploited in [8] to examine games which bridge Nim and Wythoff's game. The papers [6] and [11] are devoted to the new games obtained by adjoining to a given game $\Gamma$ its $P$-positions as additional moves, where $\Gamma$ are $a$-Wythoff's game and $(s, t)$-Wythoff's game, respectively.

The following questions 1 and 2 are nature:

Question 1. Are there a pair of games $\Gamma$ and $\Gamma_{e}$ with $\mathscr{M}(\Gamma) \subset \mathscr{M}\left(\Gamma_{e}\right)$ such that $\mathscr{P}\left(\Gamma_{e}\right) \neq \mathscr{P}(\Gamma)$, i.e., an extension of $\Gamma$ do not preserve the same set of $P$-positions of $\Gamma$ ?

Question 2. Are there a pair of games $\Gamma$ and $\Gamma_{e}$ with $\mathscr{M}(\Gamma) \subset \mathscr{M}\left(\Gamma_{e}\right)$ such that $\mathscr{P}\left(\Gamma_{e}\right)=\mathscr{P}(\Gamma)$, i.e., an extension of $\Gamma$ gives the same set of $P$-positions of $\Gamma$ ?

The answer to question 1 is ordinary. Normally, a slight change on the rule-set of $\Gamma$ would lead to the change of the whole set of $P$-positions of $\Gamma$. As an example, let $\Gamma$ be Wythoff's game. The partial result of [3] showed us that $\mathscr{P}\left(\Gamma_{r}\right) \neq \mathscr{P}(\Gamma)$ for any restriction $\Gamma_{r}$ of $\Gamma$, i.e., no strict subset of rules of Wythoff's game gives the same set of $P$-positions. Note that $\Gamma$ is an extension $\Gamma_{r}$.

Generally, under normal play convention, adjoining to Wyt $(f)$ a $P$-position do not preserve the same set of $P$-positions of $\operatorname{Wyt}(f):(0,0)$ is a $P$-position. Let $\left(A_{k}, B_{k}\right)$ with $k \geq 1$ be a $P$-position of Wyt $(f)$ and $\Gamma_{e}$ be the game obtained from $\operatorname{Wyt}(f)$ by adjoining to it the $P$-position $\left(A_{k}, B_{k}\right)$ as an additional move. Then $\left(A_{k}, B_{k}\right) \rightarrow(0,0) \in \mathscr{P}\left(\Gamma_{e}\right)$, i.e., $\left(A_{k}, B_{k}\right)$ is an $N$-position of $\Gamma_{e}$. Hence $\mathscr{P}\left(\Gamma_{e}\right)$ is not the same set of all $P$-positions of $\mathrm{Wyt}(f)$. 
The answer to question 2 is also ordinary. Let $\Gamma$ be $a$-Wythoff's game, i.e., $f(x)=x+a$ in $\operatorname{Wyt}(f)$. The authors in [6] considered three new games under normal play convention: Let $\Gamma_{1}$ (resp., $\left.\Gamma_{2}, \Gamma_{3}\right)$ be the game obtained from $\Gamma$ by adjoining to it the $P$-position $\left(A_{1}, B_{1}\right)=$ $(1, a+1)$ (resp., two $P$-positions $\left(A_{1}, B_{1}\right)$ and $\left.\left(A_{a+3}, B_{a+3}\right)\right)$, all $P$-positions $\bigcup_{n=1}^{\infty}\left\{\left(A_{n}, B_{n}\right)\right\}$ as additional moves. It turns out that $\mathscr{P}\left(\Gamma_{3}\right)=\mathscr{P}\left(\Gamma_{1}\right)$ if $a=2$, and $\mathscr{P}\left(\Gamma_{3}\right)=\mathscr{P}\left(\Gamma_{2}\right)$ if $a>2$. These two facts give a positive answer to question 2 . The fact $\mathscr{P}\left(\Gamma_{1}\right) \neq \mathscr{P}(\Gamma)$ answers affirmatively question 1 .

\subsection{Our games and results}

We consider the following ordered pure P-extension: Given a function $f$ and assume that the set of all $P$-positions of $W y t(f)$ under normal play convention is $\mathscr{P}(f)=\bigcup_{n=0}^{\infty}\left\{\left(A_{n}, B_{n}\right)\right\}$ with $\left(A_{0}, B_{0}\right)=(0,0)$. Let $K \geq 1$ be an integer. By $\Gamma_{K}$, we denote the new game obtained from Wyt $(f)$ by adjoining to it the first $K P$-positions $\bigcup_{1 \leq i \leq K}\left(A_{i}, B_{i}\right)$ as additional moves. In particular, let $\Gamma_{0}=W y t(f)$.

For an integer $m \geq 1$, if $\mathscr{P}\left(\Gamma_{m}\right) \neq \mathscr{P}\left(\Gamma_{m-1}\right)$, we call $m$ a jump-point of $\operatorname{Wyt}(f)$. Otherwise, we call $m$ a stable-point of $W y t(f)$. Obviously, $\mathscr{P}\left(\Gamma_{1}\right) \neq \mathscr{P}\left(\Gamma_{0}\right)$, i.e., 1 is a jump-point of $\operatorname{Wyt}(f)$.

The main purpose of this paper is to analyze the structure and total number of jump-point of $\mathrm{Wyt}(f)$. It is not easy to give a complete answer as the function $\mathrm{f}$ has many different forms. The authors in [6] considered the case $f(x)=x+t$ with integer $t \geq 2$. Their results showed us that 1 and $t+3$ are the only two jump-points if $t>2$, and 1 is the only jumppoint if $t=2$. The paper [11] was concerned with the question 2 for $f(x)=s x+t$. It turned out that there are many stable-points, but the total number of jump-points can not be determined. 
Let us briefly present the content of this paper. The present paper is devoted to ordered pure $P$-extensions $\Gamma_{K}$ for $f(x)=s x$ with integer coefficient $s \geq 2$, under normal play convention. Theorem 3 gives the set $\mathscr{P}\left(\Gamma_{1}\right)$ of all $P$-positions of $\Gamma_{1}$ for all integers $s \geq 2$. Theorem 4 shows us that $\mathscr{P}\left(\Gamma_{K}\right)=\mathscr{P}\left(\Gamma_{1}\right)$ for all integers $K \geq 1$ if $s=2$, i.e., 1 is the only jump-point. Theorem 6 determines the set $\mathscr{P}\left(\Gamma_{2}\right)$ of all $P$-positions of $\Gamma_{2}$ for all integers $s \geq 3$, and shows that $\mathscr{P}\left(\Gamma_{K}\right)=\mathscr{P}\left(\Gamma_{2}\right)$ for all integers $K \geq 3$ and $s \geq 3$. This fact, together with Remark 1, implies that 1 and 2 are the only two jump-points for all integers $s \geq 3$.

\section{All Jump-Points of $\Gamma_{K}$ with $f(x)=s x$}

Definition 1. Given a function $f$ and assume that the set of all $P$-positions of $\operatorname{Wyt}(f)$ is $\bigcup_{n=0}^{\infty}\left\{\left(A_{n}, B_{n}\right)\right\}$. Let $K \geq 1$ be an integer. By $\Gamma_{K}$, we denote the new game obtained from $\operatorname{Wyt}(f)$ by adjoining to Wyt $(f)$ the $P$-positions $\cup_{1 \leq i \leq K}\left(A_{i}, B_{i}\right)$ as additional moves. More clearly, three rules of moves of $\Gamma_{K}$ are allowed:

Type I. A player may remove any positive number of tokens from a single pile, possibly the entire pile.

Type II. A player may take tokens from both piles, $k>0$ from one pile and $\ell>0$ from the other. This move is restricted by the condition

$$
0<k \leq \ell<f(k) .
$$

Type III. A player takes tokens from both piles, $A_{i}$ tokens from one pile and $B_{i}$ tokens from the other, where $i \in\{1,2, \ldots, K\}$.

When analyzing the $P$-positions we frequently use the mex function: Let $S$ be a finite set of nonnegative integers. Then $\operatorname{mex}(S)$ is defined to be the least nonnegative integer not in $S$. In particular, $\operatorname{mex}(\emptyset)=0$.

In [9], the authors gave the following results: Let $f(x)=s x$ with $s \geq 2$. Under normal play convention, we have 


$$
\mathscr{P}\left(\Gamma_{0}\right)=\bigcup_{n=0}^{\infty}\left\{\left(A_{n}, B_{n}\right),\left(B_{n}, A_{n}\right)\right\},
$$

where for $n \geq 0$,

$$
\left\{\begin{array}{l}
A_{n}=\operatorname{mex}\left\{A_{i}, B_{i} \mid 0 \leq i<n\right\}, \\
B_{n}=s A_{n} .
\end{array}\right.
$$

For the case $s=1$, i.e., $f(x)=x$, the moves of Type II cannot be done and the original game reduces to Nim on Two Piles. This special case is omitted by this paper. We define two sequences $C_{n}$ and $D_{n}$ which will be used to present the formula of $\mathscr{P}\left(\Gamma_{1}\right)$.

Lemma 2. Given any $f(x)=s x$ with $s \geq 2$, we define two sequences $C_{n}$ and $D_{n}$ :

$$
\left\{\begin{array}{l}
C_{0}=D_{0}=0 \text { and for integer } n \geq 1 \\
C_{n}=\operatorname{mex}\left\{C_{i}, D_{i} \mid 0 \leq i<n\right\} \\
D_{n}=(s-1) C_{n}+2 n
\end{array}\right.
$$

Then

(1) Both $C_{n}$ and $D_{n}$ are strictly increasing sequences.

(2) $D_{n} \geq C_{n}+2 n>G_{n}$ for any integer $n \geq 1$.

(3) $C_{n}-C_{n-1} \in\{1,2\}$.

(4) $D_{n}-D_{n-1} \in\{s+1,2 s\}$. Moreover, $D_{n}-D_{n-1}=s+1$ if and only if $C_{n}-C_{n-1}=1 ; D_{n}-D_{n-1}=2 s$ if and only if $C_{n}-C_{n-1}=2$.

(5) For integers $n \geq i \geq 0$, we have $n-i \leq C_{n}-C_{i} \leq 2(n-i)$.

(6) For integers $n>i \geq 0$, we have $D_{n}-D_{i} \geq s\left(C_{n}-C_{i}\right)$.

(7) For integers $n>i \geq 0$, we have

$$
D_{n}-C_{i} \geq D_{n}-D_{i}>C_{n}-C_{i} \geq C_{n}-D_{i} .
$$


Proof. (1) By the definition of mex, $C_{n}$ is strictly increasing sequence. Also for $n \geq 1$,

$$
D_{n}-D_{n-1}=(s-1)\left(C_{n}-C_{n-1}\right)+2>0 .
$$

(2) The condition $s \geq 2$ implies that $D_{n}=(s-1) C_{n}+2 n \geq C_{n}+2 n>C_{n}$ for $n \geq 1$.

(3) By (1), $C_{n}$ is strictly increasing sequence, i.e., $C_{n}-C_{n-1}>0$. Suppose $C_{n}-C_{n-1}>2$. Then

$$
C_{n-1}<C_{n-1}+1<C_{n-1}+2<C_{n}=\operatorname{mex}\left\{C_{k}, D_{k} \mid 0 \leq k<n\right\},
$$

i.e., $C_{n-1}+1, C_{n-1}+2 \in\left\{C_{k}, D_{k} \mid 0 \leq k<n\right\}$. Then we have $C_{n-1}+1$, $C_{n-1}+2 \in \bigcup_{k=0}^{n-1} D_{k}$, i.e., there exist two integers $i, t \in\{0,1, \ldots, n-1\}$ with $i<t$ such that $D_{i}=C_{n-1}+1$ and $D_{t}=C_{n-1}+2$. Thus,

$$
1=D_{t}-D_{i}=(s-1)\left(C_{t}-C_{i}\right)+2(t-i)>(s-1)\left(C_{t}-C_{i}\right) \geq s-1 \geq 1,
$$

a contradiction. Hence $C_{n}-C_{n-1} \in\{1,2\}$.

(4) If $C_{n}-C_{n-1}=1$, then $D_{n}-D_{n-1}=(s-1)\left(C_{n}-C_{n-1}\right)+2=s+1$. If $C_{n}-C_{n-1}=2$, then $D_{n}-D_{n-1}=(s-1)\left(C_{n}-C_{n-1}\right)+2=2 s$.

(5) By (3), we have $n-i \leq C_{n}-C_{i}=\sum_{j=i+1}^{n}\left(C_{j}-C_{j-1}\right) \leq 2(n-i)$.

(6) For integers $n>i \geq 0$, by (5), we have

$$
\begin{aligned}
D_{n}-D_{i} & =(s-1) C_{n}+2 n-(s-1) C_{i}-2 i \\
& =(s-1)\left(C_{n}-C_{i}\right)+2(n-i) \\
& \geq(s-1)\left(C_{n}-C_{i}\right)+C_{n}-C_{i} \\
& =s\left(C_{s}-C_{i}\right) .
\end{aligned}
$$

(7) By (6), we have $D_{n}-D_{i} \geq s\left(C_{n}-C_{i}\right)>C_{n}-C_{i}$. Then by (2), $D_{n}-C_{i} \geq D_{n}-D_{i}>C_{n}-C_{i} \geq C_{n}-D_{i}$ for integers $n>i \geq 0$.

The proof is completed. 
Theorem 3. Given $f(x)=s x$ with $s \geq 2$ by $\mathscr{P}_{K}(s)$ we denote the set of all P-positions of $\Gamma_{K}$. Then for $K=1$,

$$
\mathscr{P}_{1}(s)=\bigcup_{n=0}^{\infty}\left\{\left(C_{n}, D_{n}\right),\left(D_{n}, C_{n}\right)\right\},
$$

where $C_{n}$ and $D_{n}$ are determined by Equation (4).

Proof. We use the notation $\left(x_{1}, y_{1}\right) \rightarrow\left(x_{2}, y_{2}\right)$ if there is a legal move from $\left(x_{1}, y_{1}\right)$ to $\left(x_{2}, y_{2}\right)$.

Proof of Fact I. Every move from any position $u \in \mathscr{P}_{1}(s)$ results in a position not in $\mathscr{P}_{1}(s)$ by any legal move of $\Gamma_{1}$. Let $u=\left(C_{n}, D_{n}\right)$ be a position in $\mathscr{P}_{1}(s)$.

(1) Suppose that $u=\left(C_{n}, D_{n}\right) \rightarrow\left(C_{i}, D_{i}\right)$ be a move of Type I.

If $C_{n}=C_{i}$ and $D_{n}>D_{i}$, then $C_{n}=C_{i}$ implies $n=i$ since $C_{n}$ is strictly increasing sequence. Thus $D_{n}=D_{i}$, which contradicts $D_{n}>D_{i}$.

If $D_{n}=D_{i}$ and $C_{n}>C_{i}$, then $D_{n}=D_{i}$ implies $n=i$ since $D_{n}$ is strictly increasing sequence. Thus $C_{n}=C_{i}$, which contradicts $C_{n}>C_{i}$.

(2) Suppose that $u=\left(C_{n}, D_{n}\right) \rightarrow\left(C_{i}, D_{i}\right)$ with $0 \leq i<n$ be a move of Type II. By Lemma 2(7), we have $D_{n}-C_{i} \geq D_{n}-D_{i}>C_{n}-C_{i} \geq C_{n}-D_{i}$ for integers $n>i \geq 0$. It suffices to consider the following two possibilities:

(2.1) $\left(C_{n}, D_{n}\right) \rightarrow\left(C_{i}, D_{i}\right)$ with $k=C_{n}-C_{i}>0$ and $\ell=D_{n}-D_{i}>0$.

Now $\ell>k>0$. By Lemma 2(6), we have $\ell=D_{n}-D_{i} \geq s\left(C_{n}-C i\right)=$ $s k=f(k)$, which contradicts $0<k \leq \ell<f(k)$.

(2.2) $\left(C_{n}, D_{n}\right) \rightarrow\left(D_{i}, C_{i}\right)$ with $k_{1}=C_{n}-D_{i}$ and $\ell_{1}=D_{n}-C_{i}$. 
By Lemma 2(6) and (7), we have $\ell_{1}>k_{1}>0$ and

$$
\ell_{1}=D_{n}-C_{i} \geq D_{n}-D_{i} \geq s\left(C_{n}-C_{i}\right) \geq s\left(C_{n}-D_{i}\right)=s k_{1}=f\left(k_{1}\right),
$$

which contradicts $0<k_{1} \leq \ell_{1}<f\left(k_{1}\right)$.

(3) Suppose that $u=\left(C_{n}, D_{n}\right) \rightarrow\left(C_{i}, D_{i}\right)$ with $0 \leq i<n$ be a move of Type III. By Equation (5) and $1=A_{1}<B_{1}=s$, we only need to consider the following two cases:

(3.1) $\left(C_{n}, D_{n}\right) \rightarrow\left(C_{i}, D_{i}\right)$ with $C_{n}-C_{i}=A_{1}$ and $D_{n}-D_{i}=B_{1}$.

It follows from Lemma 2(4) that

$$
\begin{aligned}
B_{1} & =D_{n}-D_{i}=\left(D_{n}-D_{n-1}\right)+\left(D_{n-1}-D_{n-2}\right)+\cdots+\left(D_{i+1}-D i\right) \\
& \geq(n-i)(s+1) \\
& >s=B_{1},
\end{aligned}
$$

a contradiction.

(3.2) $\left(C_{n}, D_{n}\right) \rightarrow\left(C_{i}, D_{i}\right)$ with $C_{n}-D_{i}=A_{1}$ and $D_{n}-C_{i}=B_{1}$.

It follows from Lemma 2(2) and (4) that $B_{1}=D_{n}-C_{i} \geq D_{n}-D_{i}$ $\geq(n-i)(s+1)>s=B_{1}$. This is a contradiction.

Proof of Fact II. For any position $u=(x, y) \notin \mathscr{P}_{1}(s)$, we can move from $u$ to $v \in \mathscr{P}_{1}(s)$ by a legal move of $\Gamma_{1}$.

If $y>x=0$, then we move $(x, y)=(0, y) \rightarrow(0,0) \in \mathscr{P}_{1}(s)$ by a legal move of Type I. Without loss of generality, we assume $y \geq x \geq 1$. We have either $x=C_{n}$ or $x=D_{n}$ for some integer $n \geq 1$.

Case (i) $x=D_{n}$. Now $y \geq x=D_{n}>C_{n}$, we can move $(x, y)=$ $\left(D_{n}, y\right) \rightarrow\left(D_{n}, C_{n}\right) \in \mathscr{P}_{1}(s)$ by taking $y-C_{n}>0$ tokens from the heap of size $y$. 
Case (ii) $x=C_{n}$. Now $y \neq D_{n}$. We consider four following possibilities: (1) $y>D_{n}$; (2) $y=D_{n}-1$; (3) $s C_{n} \leq y<D_{n}-1$; (4) $C_{n}=$ $x \leq y<s C_{n}$.

(1) $y>D_{n}$. We move $(x, y)=\left(C_{n}, y\right) \rightarrow\left(C_{n}, D_{n}\right) \in \mathscr{P}_{1}(s)$, by a legal move of Type I.

(2) $y=D_{n}-1$. Now we move

$$
(x, y)=\left(C_{n}, D_{n}-1\right) \rightarrow\left(C_{n-1}, D_{n-1}\right) \in \mathscr{P}_{1}(s),
$$

with $k=C_{n}-C_{n-1}$ and $\ell=D_{n}-1-D_{n-1}$. This is a legal move. Indeed,

$$
\ell=D_{n}-1-D_{n-1}=(s-1)\left(C_{n}-C_{n-1}\right)+1=(s-1) k+1 .
$$

By Lemma 2(3), $k=C_{n}-C_{n-1} \in\{1,2\}$. If $k=1=A_{1}$, then $\ell=s=B_{1}$, giving a legal move of Type III. If $k=2$, then $k=2<2 s-1=\ell<2 s=f(k)$, giving a legal move of Type II.

(3) $s C_{n} \leq y<D_{n}-1$. Now we move

$$
(x, y)=\left(C_{n}, y\right) \rightarrow\left(C_{i}, D_{i}\right) \in \mathscr{P}_{1}(s),
$$

where $k=C_{n}-C_{i}$ and $\ell=y-D_{i}$ and $i=y-(s-1) C_{n}-n+1$. This is a legal move of Type II: Note that $i \geq C_{n}-n+1 \geq 1$.

(a) $k>0$. Indeed, $y<D_{n}-1$ implies that $i<D_{n}-1-(s-1) C_{n}-n$ $+1=n$. Thus $k=C_{n}-C_{i}>0$.

(b) $\ell \geq k$. By the definition of $i$, we have $y=(s-1) C_{n}+n+i-1$. Thus,

$$
\ell=y-D_{i}=(s-1)\left(C_{n}-C_{i}\right)+n-i-1 \geq C_{n}-C_{i}=k .
$$

(c) $\ell<s k$. It follows from Lemma 2(5) that

$$
\begin{aligned}
\ell & =y-D_{i}=(s-1)\left(C_{n}-C_{i}\right)+n-i-1 \\
& \leq(s-1)\left(C_{n}-C_{i}\right)+\left(C_{n}-C_{i}\right)-1 \\
& <s k .
\end{aligned}
$$


(4) $C_{n}=x \leq y<s C_{n}$. We move $(x, y)=\left(C_{n}, y\right) \rightarrow(0,0) \in \mathscr{P}_{1}(s)$, by a legal move of Type II: $0<k=C_{n} \leq \ell=y<s C_{n}=f(k)$.

The proof is complete.

Theorem 4. Given $f(x)=s x$ with $s=2$, by $\mathscr{P}_{K}(s)$ we denote the set of all P-positions of $\Gamma_{K}$. Then for all integers $K \geq 2$,

$$
\mathscr{P}_{K}(s)=\mathscr{P}_{1}(s),
$$

where $\mathscr{P}_{1}$ is determined by Equations (6) and (4).

Proof. Recall that $\Gamma_{K}$ is obtained by adjoining to $\Gamma_{1}$ the moves $\bigcup_{i=2}^{K}\left(A_{i}, B_{i}\right)$. We show that the addition of these moves leaves the $P$-positions of $\Gamma_{1}$ invariant. The proof is based on Theorem 3.

Proof of Fact I. Let $\left(C_{n}, D_{n}\right)$ be a position in $\mathscr{P}_{1}(s)$, the proof of Fact $\mathrm{I}$ in Theorem 3 implies that $u=\left(C_{n}, D_{n}\right)$ lands in a position not in $\mathscr{P}_{1}(s)$ by any legal move of Types I or II, or the move $\left(A_{1}, B_{1}\right)=(1,2)$ of Type III.

It suffices to show that the move $\left(C_{n}, D_{n}\right) \rightarrow\left(C_{i}, D_{i}\right)$, for every $n>i \geq 0$, can not be $\left(A_{k}, B_{k}\right)$ for any integer $k \in\{2,3, \ldots, K\}$ :

Case 1. Suppose that there exists an integer $k \geq 2$ such that $A_{k}=C_{n}-C_{i}$ and $B_{k}=D_{n}-D_{i}$. Let $C=\bigcup_{j=1}^{\infty}\left\{C_{j}\right\}, D=\bigcup_{j=1}^{\infty}\left\{D_{j}\right\}$. By $\sharp(U)$, we denote the number of elements in a given set $U$. We define

$$
\left\{\begin{array}{l}
S=\left\{x \mid x \text { is an integer and } C_{i}<x \leq C_{n}\right\}, \\
n_{C}=\sharp(S \cap C), \\
n_{D}=\sharp(S \cap D) .
\end{array}\right.
$$

Then $\sharp(S)=n_{C}+n_{D}, \sharp(S)=C_{n}-C_{i}=A_{k}$ and $n_{C}=n-i$. Thus

$$
n-i=A_{k}-n_{D} .
$$


Let $r:=n_{D}$ be the number of elements in $D$ between $C_{i}$ and $C_{n}$, i.e., $A_{k}=n-i+r$.

By the definitions of $A_{n}$ and $B_{n}, A_{0}=B_{0}=0, A_{1}=1, B_{1}=s=2$. Thus $A_{k} \geq A_{2}=\operatorname{mex}\{0,1,2\}=3$ for any integer $k \geq 2$. If $n-i=1,3 \leq$ $A_{k}=C_{n}-C_{i}=C_{i+1}-C_{i} \leq 2$, a contradiction. If $n-i>1$, there are $D_{j+1}, D_{j+2}, \ldots, D_{j+r}$ between $C_{i}$ and $C_{n}$ such that $D_{j+1} \geq C_{i}+1$ and $D_{j+r} \leq C_{n}-1$. Then,

$$
D_{j+r}-D_{j+1} \leq C_{n}-1-\left(C_{i}+1\right)=A_{k}-2=n-i+r-2 .
$$

On the other hand, by Lemma 2(4), we have $D_{j+r}-D_{j+1} \geq(r-1)(s+1)$ $=3(r-1)$. Hence $3(r-1) \leq n-i+r-2$, i.e., $r<n-i$.

By Lemma 2(4), we have

$$
\begin{aligned}
D_{n}-D_{i} & =\left(D_{n}-D_{n-1}\right)+\left(D_{n-1}-D_{n-2}\right)+\ldots+\left(D_{i+1}-D_{i}\right) \\
& =(n-i-r)(s+1)+2 r s \\
& =(n-i+r) s+n-i-r \\
& =s A_{k}+n-i-r \\
& >s A_{k}=B_{k},
\end{aligned}
$$

which contradicts $B_{k}=D_{n}-D_{i}$.

Case 2. Suppose that $A_{k}=C_{n}-D_{i}$ and $B_{k}=D_{n}-C_{i}$ for any $n>i \geq 1$. By Lemma 2(6) and (7), we have $D_{n}-C_{i}>D_{n}-D_{i} \geq s$ $\left(C_{n}-C_{i}\right) \geq s\left(C_{n}-C_{i}\right)=s A_{k}=B_{k}$, which contradicts $B_{k}=D_{n}-C_{i}$.

Proof of Fact II. Let $u$ be a position which is not in $\mathscr{P}_{1}(s)$. The proof of Fact II in Theorem 3 implies that we can move from $u$ to $v \in \mathscr{P}_{1}(s)$ by a legal move of $\Gamma_{1}$. We note that the set of legal moves of $\Gamma_{1}$ is a subset of legal moves of $\Gamma_{K}$. Hence, we can move from $u$ to $v \in \mathscr{P}_{1}(s)$ by a legal move of $\Gamma_{K}$. 
The proof is completed.

Remark 1. If $f(x)=s x$ with $s \geq 3$, it follows from Equation (3) that $A_{0}=B_{0}=0, A_{1}=1, B_{1}=s \geq 3$. Thus $A_{k} \geq A_{2}=\operatorname{mex}\left\{0,1, B_{1}\right\}=2$ for $k \geq 2$. In the proof of Case 1 in Theorem 4, if $n-i=1$, then $2=A_{2}=n-i+r$ implies $r=1$, i.e., there exists an integer $j$ such that $C_{i}<D_{j}<C_{i+1}$. Thus $C_{i+1}-C_{i}=2=A_{2}$ and

$$
D_{i+1}-D_{i}=(s-1)\left(C_{i+1}-C_{i}\right)+2=2 s=f\left(A_{2}\right)=B_{2} .
$$

Hence adjoining $\left(A_{2}, B_{2}\right)$ to $\Gamma_{1}$ will change the set $\mathscr{P}_{1}(s)$.

Theorem 6 presents the explicit formula of $\mathscr{P}_{2}(s)$ for all integers $s \geq 3$ by two new sequences $G_{n}$ and $H_{n}$ defined in Lemma 5 . Moreover, Theorem 6 shows that $\mathscr{P}_{K}(s)=\mathscr{P}_{2}(s)$ for all integers $K \geq 3$ and $s \geq 3$.

Lemma 5. Given $f(x)=s x$ with $s \geq 3$, we define two sequences $G_{n}$ and $H_{n}$ :

$$
\left\{\begin{array}{l}
G_{0}=H_{0}=0 \text { and for integer } n \geq 1 \\
G_{n}=\operatorname{mex}\left\{G_{i}, H_{i} \mid 0 \leq i<n\right\} \\
H_{n}=s G_{n}+n
\end{array}\right.
$$

Then

(1) Both $G_{n}$ and $H_{n}$ are strictly increasing sequences.

(2) $H_{n}>G_{n}$ for integer $n \geq 1$.

(3) $G_{n}-G_{n-1} \in\{1,2\}$.

(4) $H_{n}-H_{n-1} \in\{s+1,2 s+1\}$. Moreover, $H_{n}-H_{n-1}=s+1$ if and only if $G_{n}-G_{n-1}=1 ; H_{n}-H_{n-1}=2 s+1$ if and only if $G_{n}-G_{n-1}=2$.

(5) For integers $n>i \geq 0$, we have

$$
H_{n}-G_{i} \geq H_{n}-H_{i}>G_{n}-G_{i} \geq G_{n}-H_{i} .
$$


Proof. (1) By the definition of mex, $G_{n}$ is strictly increasing sequence. Also for $n \geq 1, H_{n}-H_{n-1}=s\left(G_{n}-G_{n-1}\right)+1>0$.

(2) The condition $s \geq 3$ gives $H_{n}=s G_{n}+n>G_{n}$ for $n \geq 1$.

(3) By (1), $G_{n}$ is strictly increasing sequence, i.e., $G_{n}-G_{n-1}>0$. If $G_{n}-G_{n-1}>2$, then

$$
G_{n-1}<G_{n-1}+1<G_{n-1}+2<G_{n}=\operatorname{mex}\left\{G_{k}, H_{k} \mid 0 \leq k<n\right\},
$$

i.e., $G_{n-1}+1, G_{n-1}+2 \in\left\{G_{k}, H_{k} \mid 0 \leq k<n\right\}$. Then $G_{n-1}+1, G_{n-1}+2$ $\in \bigcup_{k=0}^{n-1} H_{k}$, i.e., there exist two integers $i, t \in\{0,1, \ldots, n-1\}$ with $t>i$ such that $H_{i}=G_{n-1}+1$ and $H_{t}=G_{n-1}+2$. Now, $1=H_{t}-H_{i}=$ $s\left(G_{t}-G_{i}\right)+(t-i) \geq s+1>1$, giving a contradiction. Hence, $G_{n}-G_{n-1}$ $\in\{1,2\}$.

(4) If $G_{n}-G_{n-1}=1$, then $H_{n}-H_{n-1}=s\left(G_{n}-G_{n-1}\right)+1=s+1$. If $G_{n}-G_{n-1}=2$, then $H_{n}-H_{n-1}=s\left(G_{n}-G_{n-1}\right)+1=2 s+1$.

(5) The condition $s \geq 3$ gives $H_{n}-H_{i}=s\left(G_{n}-G_{i}\right)+(n-i)>G_{n}-G_{i}$. By (2), we have $H_{n}-G_{i} \geq H_{n}-H_{i}>G_{n}-G_{i} \geq G_{n}-H_{i}$ for integers $n>i \geq 0$.

The proof is completed.

Theorem 6. Given $f(x)=s x$ with $s \geq 3$, by $\mathscr{P}_{K}(s)$ we denote the set of all P-positions of $\Gamma_{K}$. Then

(A) For $K=2$ and $s \geq 3$,

$$
\mathscr{P}_{2}(s)=\bigcup_{n=0}^{\infty}\left\{\left(G_{n}, H_{n}\right),\left(H_{n}, G_{n}\right)\right\},
$$

where $G_{n}$ and $H_{n}$ are determined by Equation (8). 
(B) For $K \geq 3$ and $s \geq 3$,

$$
\mathscr{P}_{K}(s)=\mathscr{P}_{2}(s)
$$

where $\mathscr{P}_{2}(s)$ is determined by Equation (10).

\section{Proof of (A).}

(A.1) Every move from any position $u \in \mathscr{P}_{2}(s)$ results in a position not in $\mathscr{P}_{2}(s)$ by any legal move of $T_{2}$. Let $u=\left(G_{n}, H_{n}\right)$ be a position in $\mathscr{P}_{2}(s)$.

(1) Similar to the proof of Fact I of Theorem 3, Lemma 5(1) means that $u=\left(G_{n}, H_{n}\right) \rightarrow\left(G_{i}, H_{i}\right)$ can not be a legal move of Type I.

(2) Suppose that $u=\left(G_{n}, H_{n}\right) \rightarrow\left(G_{i}, H_{i}\right)$ with $0 \leq i<n$ be a move of Type II. By Lemma 5(5), we have $H_{n}-G_{i} \geq H_{n}-H_{i}>G_{n}-G_{i} \geq$ $G_{n}-H_{i}$ for integers $n>i \geq 0$. So, we need only consider the following two possibilities:

(2.1) $\left(G_{n}, H_{n}\right) \rightarrow\left(G_{i}, H_{i}\right)$ with $k=G_{n}-G_{i}>0$ and $\ell=H_{n}-H_{i}>0$.

Then

$$
\ell=H_{n}-H_{i}=s\left(G_{n}-G_{i}\right)+(n-i)>s\left(G_{n}-G_{i}\right)=s k=f(k)>k,
$$

which contradicts $0<k \leq l<f(k)$.

(2.2) $\left(G_{n}, H_{n}\right) \rightarrow\left(H_{i}, G_{i}\right)$ with $k_{2}=G_{n}-H_{i}$ and $\ell_{2}=H_{n}-G_{i}$. It follows from Lemma 5(2) and Equation (12) that

$$
\ell_{2}=H_{n}-G_{i} \geq H_{n}-H_{i}>s\left(G_{n}-G_{i}\right) \geq s\left(G_{n}-H_{i}\right)=s k_{2}=f\left(k_{2}\right)>k_{2},
$$

which contradicts $0<k_{2} \leq \ell_{2}<f\left(k_{2}\right)$.

(3) Suppose that $u=\left(G_{n}, H_{n}\right) \rightarrow\left(G_{i}, H_{i}\right)$ with $0 \leq i<n$ be a move of Type III: $\left(A_{1}, B_{1}\right)$ or $\left(A_{2}, B_{2}\right)$. 
By Equation (9) and $A_{1}<B_{1}$ and $A_{2}<B_{2}$, we need only consider two cases:

(3.1) $A_{j}=G_{n}-G_{i}$ and $B_{j}=H_{n}-H_{i}$ for some $j \in\{1,2\}$. Then $B_{j}=H_{n}-H_{i}=s\left(G_{n}-G_{i}\right)+(n-i)>s\left(G_{n}-G_{i}\right)=s A_{j}=B_{j}$, giving a contradiction.

(3.2) $A_{j}=G_{n}-H_{i}$ and $B_{j}=H_{n}-G_{i}$ for some $j \in\{1,2\}$. Then $B_{j}=H_{n}-G_{i} \geq H_{n}-H_{i}>s\left(G_{n}-G_{i}\right) \geq s\left(G_{n}-H_{i}\right)=s A_{j}=B_{j}$. This is a contradiction.

(A.2) For any position $u=(x, y) \notin \mathscr{P}_{2}(s)$, we can move from $u$ to $v \in \mathscr{P}_{2}(s)$ by a legal move of $\Gamma_{2}$.

Without loss of generality, we assume $0 \leq x \leq y$. If $y>x=0$, then we move $(x, y)=(0, y) \rightarrow(0,0) \in \mathscr{P}_{2}(s)$ by a legal move of Type I. For $y \geq x \geq 1$, we have either $x=G_{n}$ or $x=H_{n}$ for some integer $n \geq 1$.

Case (i) $x=H_{n}$. Now $y \geq x=H_{n}>G_{n}$. We move $(x, y)=\left(H_{n}, y\right)$ $\rightarrow\left(H_{n}, G_{n}\right) \in \mathscr{P}_{2}(s)$, by taking $y-G_{n}>0$ tokens from the heap of size $y$.

Case (ii) $x=G_{n}$. Now $y \neq H_{n}$ and we proceed by distinguishing four possibilities: (1) $y>H_{n}$; (2) $y=H_{n}-1$; (3) $s G_{n} \leq y<H_{n}-1$; (4) $G_{n}=x \leq y<s G_{n}$.

(1) $y>H_{n}$. We can move $(x, y)=\left(G_{n}, y\right) \rightarrow\left(G_{n}, H_{n}\right) \in \mathscr{P}_{2}(s)$ by taking $y-H_{n}>0$ tokens from the heap of size $y$.

(2) $y=H_{n}-1$. We move

$$
(x, y)=\left(G_{n}, H_{n}-1\right) \rightarrow\left(G_{n-1}, H_{n-1}\right) \in \mathscr{P}_{2}(s),
$$

with $k=G_{n}-G_{n-1}$ and $\ell=H_{n}-1-H_{n-1}$. This is a legal move of Type III: 
Indeed, $\quad \ell=H_{n}-1-H_{n-1}=s\left(G_{n}-G_{n-1}\right)=s k$. By Lemma 5(3), $k=G_{n}-G_{n-1} \in\{1,2\}$. If $k=1$, then $k=A_{1}$ and $\ell=s=B_{1}$. If $k=2$, then $k=A_{2}$ and $\ell=2 s=B_{2}$. Both are legal moves of Type III.

(3) $s G_{n} \leq y<H_{n}-1$. We move

$$
(x, y)=\left(G_{n}, y\right) \rightarrow\left(G_{i}, H_{i}\right) \in \mathscr{P}_{2}(s),
$$

where $k=G_{n}-G_{i}$ and $\ell=y-H_{i}$ and $i=y-s G_{n}+1$. We will show that this is a legal move of Type II. Note that $i=y-s G_{n}+1 \geq 1>0$. It suffices to check the following facts:

(a) $k>0$. Indeed, $y<H_{n}-1$ implies that $i=y-s G_{n}+1<H_{n}-1$ $-s G_{n}+1=n$. Thus $k=G_{n}-G_{i}>0$.

(b) $\ell \geq k$. Now $y=s G_{n}-1+i$. Then $\ell=y-H_{i}=s G_{n}-1+i-\left(s G_{i}+i\right)$ $=s\left(G_{n}-G_{i}\right)-1 \geq 3 k-1 \geq k$.

(c) $\ell<s k$. By $(b)$, we have $\ell=s\left(G_{n}-G_{i}\right)-1<s k$.

(4) $G_{n}=x \leq y<s G_{n}$. We move $(x, y)=\left(G_{n}, y\right) \rightarrow(0,0) \in \mathscr{P}_{2}(s)$, by a legal move of Type II: $0<k=G_{n} \leq \ell=y<s G_{n}=f(k)$.

Proof of (B). $\Gamma_{K}$ is obtained by adjoining to $\Gamma_{2}$ the moves $\bigcup_{i=3}^{K}\left(A_{k}, B_{k}\right)$. We show that the addition of these moves leaves the $P$-positions of $\Gamma_{2}$ invariant. The proof is based on Theorem 6(A).

(B.1) Every move from any position $u \in \mathscr{P}_{2}(s)$ results in a position not in $\mathscr{P}_{2}(s)$ by any legal move of $\Gamma_{K}$.

Let $\left(G_{n}, H_{n}\right)$ be a position in $\mathscr{P}_{2}(s)$, the proof of (A.1) in Theorem 6 implies that $u=\left(G_{n}, H_{n}\right)$ lands in a position not in $\mathscr{P}_{2}(s)$ by any legal move of Types I or II, or two moves $\left(A_{1}, B_{1}\right)$ and $\left(A_{2}, B_{2}\right)$ of Type III. It suffices to show that the move $\left(G_{n}, H_{n}\right) \rightarrow\left(G_{i}, H_{i}\right)$, for every $n>i \geq 0$, can not be $\left(A_{k}, B_{k}\right)$ for any integer $k \in\{3,4, \ldots, K\}$. 
Suppose that there exists an integer $k \in\{3,4, \ldots, K\}$ such that $A_{k}=G_{n}-G_{i}$ and $B_{k}=H_{n}-H_{i}$. Then $B_{k}=H_{n}-H_{i}=s\left(G_{n}-G_{i}\right)+$ $(n-i)>s\left(G_{n}-G_{i}\right)=s A_{k}=B_{k}$. This is a contradiction.

Suppose that there exists an integer $k \in\{3,4, \ldots, K\}$ such that $A_{k}=G_{n}-H_{i}$ and $B_{k}=H_{n}-G_{i}$. By Lemma 5(2), we have $B_{k}=H_{n}-$ $G_{i} \geq H_{n}-H_{i}>s\left(G_{n}-G_{i}\right) \geq s\left(G_{n}-H_{i}\right)=s A_{k}=B_{k}$. This is another contradiction.

(B.2) For any position $u=(x, y) \notin \mathscr{P}_{2}(s)$, we can move from $u$ to $v \in \mathscr{P}_{2}(s)$ by a legal move of $\Gamma_{K}$. Let $u$ be a position which is not in $\mathscr{P}_{2}(s)$. The proof of (A.2) in Theorem 6 implies that we can move from $u$ to $v \in \mathscr{P}_{2}(s)$ by a legal move of $\Gamma_{2}$. We note that the set of legal moves of $\Gamma_{2}$ is a subset of legal moves of $\Gamma_{K}$. Hence, we can move from $u$ to $v \in \mathscr{P}_{2}(s)$ by a legal move of $\Gamma_{K}$.

The proof is completed.

\section{Acknowledgements}

The research is supported by the National Natural Science Foundation of China under Grants 11171368 and 11171094.

\section{References}

[1] E. R. Berlekamp, J. H. Conway and R. K. Guy, Winning Ways for Your Mathematical Plays, A. K. Peters, Wellesley, MA, 2004.

[2] J. H. Conway, On Numbers and Games, CRC Press, 2000.

[3] E. Duchêne, A. S. Fraenkel, R. J. Nowakowski and M. Rigo, Extensions and restrictions of Wythoff's game preserving its P-positions, J. Combin. Theory Ser. A 117 (2010), 545-567.

[4] A. S. Fraenkel, Scenic trails ascending from sea-level Nim to alpine chess, in: R. J. Nowakowski (Ed.), Games of No Chance, Proc. MSRI Workshop on Combinatorial Games, July, 1994, Berkely, CA, MSRI Publ., Vol. 29, Cambridge University Press, Cambridge, (1996), 13-42. 
[5] A. S. Fraenkel, Heap games, numeration systems and sequences, Ann. Comb. 2 (1998), 197-210.

[6] A. S. Fraenkel and M. Ozery, Adjoining to Wythoff's game its $P$-positions as moves, Theoret. Comput. Sci. 205 (1998), 283-296.

[7] A. S. Fraenkel, Wythoff games, continued fractions, cedar trees and Fibonacci searches, Theoret. Comput. Sci. 29(1-2) (1984), 49-73.

[8] A. S. Fraenkel and M. Lorberbom, Nimhoff games, J. Combin. Theory Ser. A 58 (1991), 1-25.

[9] A. S. Fraenkel and Y. Tanny, A class of Wythoff-like games, Proc. INTEGERS Conference, Carrollton, Georgia, Oct. 26-29, 2011, in: INTEGERS, Electr. J. Combinat. Number Theory 12(B) (2013), $\sharp$ A7.

[10] W. A. Liu, Y. Wang and N. Li, The $(s, t)$-Wythoff's game under misère play convention, Fifth International Joint Conference on Computational Sciences and Optimization, Harbin, China (2012), 419-422.

[11] W. A. Liu and X. Zhao, Adjoning to $(s, t)$-Wythoff's game its $P$-positions as moves, Discret. Appl. Math. 179 (2014), 28-43.

[12] W. Wythoff, A modification of the game of Nim, Nieuw Arch. Wiskd. 7 (1907), 199-202. 